\title{
Berberine ameliorates nonalcoholic fatty liver disease by decreasing the liver lipid content via reversing the abnormal expression of MTTP and LDLR
}

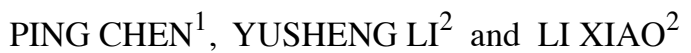 \\ ${ }^{1}$ Department of Pharmacy, Affiliated Hospital of Shandong Medical College; \\ ${ }^{2}$ Department of Pharmacy, Linyi Maternal and Child Health Care Hospital, Linyi, Shandong 276000, P.R. China
}

Received April 16, 2020; Accepted February 15, 2021

DOI: $10.3892 /$ etm.2021.10543

\begin{abstract}
The global incidence of nonalcoholic fatty liver disease (NAFLD) is increasing. The present study explored the effect and mechanism of berberine (BBR) on NAFLD in rats. Thirty-five Sprague-Dawley rats were randomly divided into the control and NAFLD groups, which were fed a normal diet or high-fat diet, respectively, for 8 weeks. Hematoxylin and eosin staining was performed on liver tissues and establishment of the NAFLD model was confirmed by microscopy. NAFLD rats were subsequently randomly subdivided and treated with saline or BBR for 8 weeks. The liver wet weight of rats in each group was measured, the liver tissue structure was observed by microscopy, and alanine aminotransferase (ALT), aspartate aminotransferase (AST), total cholesterol (TC), triglyceride (TG), fasting blood glucose (FBG), low-density lipoprotein (LDL) and high-density lipoprotein (HDL) levels were detected using a semi-automatic biochemical detector. Reverse transcription-quantitative PCR and western blotting were performed to determine the mRNA and protein expression levels of microsomal triglyceride transfer protein (MTTP), apolipoprotein B and low-density lipoprotein receptor (LDLR). Compared with the control group, the liver wet weight of the NAFLD rats was higher; the liver showed obvious fatty degeneration and liver TG levels increased significantly, as did serum levels of ALT, AST, TC, TG, FBG, HDL and LDL, while expression of MTTP and LDLR significantly decreased. Compared with the saline-treated NAFLD rats, the BBR-treated rats had reduced liver wet weight, improved liver steatosis and a significant decrease in liver TG levels, while ALT, AST, TC, TG, and LDL serum levels significantly
\end{abstract}

Correspondence to: Professor Li Xiao, Department of Pharmacy, Linyi Maternal and Child Health Care Hospital, 1 Qinghe South Road, Linyi, Shandong 276000, P.R. China

E-mail: xiao_10304@163.com

Key words: nonalcoholic fatty liver disease, berberine, microsomal triglyceride transfer protein, apolipoprotein B, low-density lipoprotein receptor decreased and MTTP levels were significantly upregulated. In conclusion, BBR treatment ameliorated the fatty liver induced by a high-fat diet in rats. Furthermore, BBR reversed the abnormal expression of MTTP and LDLR in rats with high-fat diet induced-NAFLD. The present findings suggest that fatty liver could be improved by BBR administration, via reversing the abnormal expression of MTTP and LDLR and inhibiting lipid synthesis.

\section{Introduction}

Nonalcoholic fatty liver disease (NAFLD) is the most common chronic liver disease in China (incidence 15\%). Improved quality of life has altered the diet of the Chinese. The total calorie intake has increased greatly, and a lack of effective exercise has led to an increase in the prevalence of NAFLD (1). NAFLD is a metabolic syndrome characterized by excessive accumulation of fat in hepatocytes and by hepatic parenchymal cell degeneration as a major characteristic of liver damage, which may progress to liver fibrosis, cirrhosis, and liver cancer (2). NAFLD is prone to insulin resistance and is closely related to type 2 diabetes $(3,4)$. Regarding the pathogenesis of NAFLD, the secondary percussion hypothesis is accepted. The primary cause of NAFLD is excessive deposition of triglycerides in the liver because of decreased very-low-density lipoprotein (VLDL) output and increased fatty acid oxidation $(5,6)$. NAFLD is treated by changing lifestyle factors, such as diet and exercise $(7,8)$. However, patients with poor compliance show no significant improvement, and there is no drug for NAFLD. The drugs commonly used for NAFLD include insulin sensitizers and antioxidants; however, their long-term efficacy is unclear, and new interventions for NAFLD are needed.

Berberine (BBR) has a phenyltetrahydroxyquinoline structure. Berberine bioactive alkali has the effect of improving fatty liver in mice (9). Berberine is an important component of the biologically active alkali of Coptidis Rhizoma. Previous studies have found that BBR also lowers blood sugar, corrects blood lipid disorders, reduces the production of inflammatory factors, and inhibits the release of endotoxin. It has certain pharmacological effects on metabolic syndrome, digestive-system diseases, cardiovascular diseases, tumors, 
and mental disorders (10). BBR has been used to treat type 2 diabetes (11). Because there is no drug for NAFLD, and BBR has hypoglycemic, lipid-lowering, and antioxidant effects, it has potential for the treatment of NAFLD. Xing et al (12) and Yang et al (13) reported that BBR has a protective effect on NAFLD. Other previous studies also demonstrated that BBR had an important role in NAFLD and hepatic inflammations, such as hepatic steatosis, through regulating insulin receptor substrate 2, triggering AMP-activated protein kinase and inhibiting oxidative stress (14-16). In liver lipid metabolism, the key factors for VLDL transport are microsomal triglyceride transfer protein (MTTP), apolipoprotein B (ApoB) and low-density lipoprotein receptor (LDLR), and their expression levels affect the assembly and secretion of VLDL. Pan and Hussain (17) showed that the MTTP gene was associated with the risk of NAFLD; therefore, expression of these key genes is important for BBR intervention in NAFLD. The present study established a NAFLD animal model, observed changes in liver tissue by hematoxylin and eosin (H\&E) staining, and assayed biochemical markers to evaluate the effect of berberine on NAFLD. Reverse transcription-quantitative PCR and western blotting were performed to assess the mRNA and protein expression levels of MTTP, ApoB and LDLR in liver tissue, and to explore the mechanism underlying the effect of BBR on NAFLD.

\section{Materials and methods}

Animal model of NAFLD. A rat model of NAFLD was established as described previously (18). Thirty-five specific-pathogen-free 8-week-old male SD rats (weight range, 310-322 g) were purchased from the Laboratory Animal Center of Guangzhou University of Chinese Medicine [approval no. SYXK (Yue) 2013-0034] and adaptively fed for 1 week. The rats were then randomly divided into a control group which was fed with a normal diet (ND, $n=12$; diet purchased from the Laboratory Animal Center of Jinan University) and an experimental group which was fed a high-fat diet (HFD, $n=23$; diet composed of $80 \%$ regular chow, $8 \%$ yolk powder, $10 \%$ lard oil, $1.5 \%$ cholesterol, and $0.5 \%$ bile salt; purchased from Guangdong Medical Laboratory Animal Center) for 8 weeks. Both groups had free access to the specified diet and water. The body weight of the rats was measured once weekly, and their hair color, appetite, action, feces, and response to external stimuli were observed. After 8 weeks, two rats in the ND group and three in the HFD group were euthanized by cervical dislocation under deep anesthesia (3\% pentobarbital sodium; $30 \mathrm{mg} / \mathrm{kg}$ ), and liver specimens were stained with H\&E to confirm the establishment of the model. The standard for success was liver cells with particle infiltration, and abnormal liver-function indicators. The formula for both diets is listed in Table I. All experimental procedures were approved by the Animal Experimental Ethics Committee of the Affiliated Hospital of Shandong Medical College (permit no. AEECAHSMC20205059).

$B B R$ treatment. The remaining NAFLD rats $(n=20)$ were randomly divided into a BBR intervention group $(B B R, n=10)$ and a solvent control group (HFD, $\mathrm{n}=10)$. The BBR group was intragastrically administered with $100 \mathrm{mg} / \mathrm{kg} /$ day of BBR
(Mysun Pharma Co., Ltd.) in $0.5 \%$ carmellose sodium (CS) as solvent, once daily for 8 weeks. The ND and HFD groups were only administered with the vehicle control, $4 \mathrm{ml} / \mathrm{kg} /$ day of $0.5 \%$ CS. Previous studies have used 100 and $300 \mathrm{mg} / \mathrm{kg} / \mathrm{day}$ of BBR; preliminary experiments were performed with both doses and no significant difference was observed, therefore the $100 \mathrm{mg} / \mathrm{kg} /$ day dose of BBR was selected for the present study (19-21).

Sample collection. The rats were fasted for $12 \mathrm{~h}$ prior to specimen collection. Sample collection was performed prior to the measurement detections. After anesthesia with $3 \%$ pentobarbital sodium $(30 \mathrm{mg} / \mathrm{kg})$, the rats were dissected under aseptic conditions, and $10 \mathrm{ml}$ of fresh blood was taken from the abdominal aorta (the rats that were not involved with sample collections were not anesthetized). Full anesthesia was confirmed by corneal or pedal reflex (firm toe pinch). The serum was separated at room temperature for $20 \mathrm{~min}$, followed by ultra-low-temperature centrifugation $\left(4^{\circ} \mathrm{C}\right.$, $1,006 \times \mathrm{g}, 15 \mathrm{~min})$. The supernatant was stored at $-80^{\circ} \mathrm{C}$ for later use. The liver was quickly dissected and rinsed with physiological saline. Surface moisture was blotted with filter paper, and the liver was weighed. A small piece of tissue from the right hepatic liver tip was placed in $10 \%$ formalin and fixed at room temperature for $24 \mathrm{~h}$, for pathological analysis. Sample collection was performed after rats were anaesthetized and before they were euthanized. The rats were then sacrificed by cervical dislocation at the same time. Animal death was defined as mydriasis, respiratory arrest and cardiac arrest for a period of $>5 \mathrm{~min}$.

Serum biochemical indicator detection. The biochemical indicators serum alanine aminotransferase (ALT), aspartate aminotransferase (AST), total cholesterol (TC), triglyceride (TG), fasting blood glucose (FBG), low-density lipoprotein (LDL) and high-density lipoprotein (HDL) were assayed using a 7180 model automatic biochemical analyzer (Hitachi, Ltd.).

Assessment of pathological changes in the liver by $H \& E$ staining. The specimens were fixed in $4 \%$ paraformaldehyde and embedded in paraffin. Sections were cut at $5 \mu \mathrm{m}$ thickness in the coronal plane through the repaired tendon-bone interface. The sections were stained with $\mathrm{H} \& \mathrm{E}$ and observed under a light microscope (Zeiss AG). Liver histopathological changes were scored by the NAFLD activity score (NAS) (22): steatosis (0-3 points), hepatic lobular inflammation (0-3 points), and vacuolar-like degeneration (0-2 points). Higher scores mean increased severity.

Determination of liver TG levels. Liver tissue (100 mg) was ground in chloroform: methanol (2:1 volume ratio) solution and vigorously shaken at room temperature for $2 \mathrm{~h}$. Then, $0.5 \mathrm{mg}$ of $0.1 \mathrm{M} \mathrm{NaCl}$ was added, and the mixture was centrifuged at $1530 \mathrm{x} \mathrm{g}$ at $37^{\circ} \mathrm{C}$ for $10 \mathrm{~min}$. The organic lipid oil phase was removed, and the TG levels were measured using an automatic biochemical analyzer (Hitachi, Ltd.).

Reverse transcription-quantitative PCR (RT-qPCR). qPCR analysis was performed to determine the MTTP, ApoB and 
LDLR mRNA expression levels. Total RNA from liver tissue was extracted using TRIzol reagent (Invitrogen; Thermo Fisher Scientific, Inc.), according to the manufacturer's instructions. RNA (1 $\mu \mathrm{g})$ was subjected to reverse transcription using a PrimeScript RT Reagent kit (Takara Bio, Inc.). The reverse transcription conditions were: one cycle at $37^{\circ} \mathrm{C}$ for $15 \mathrm{~min}$ and one cycle at $85^{\circ} \mathrm{C}$ for $5 \mathrm{sec}$. Next, the diluted cDNA $(2 \mu \mathrm{l})$ was subjected to qPCR using SYBR-Green I (Takara Bio, Inc.) with primers synthesized by Nanjing GenScript Co., Ltd. The mRNA expression levels were normalized to the housekeeping gene $\beta$-actin. The primer sequences were: MTTP, forward 5'-TCGGGTGGCTGTGGTAATAAC-3' and reverse 5'-AACTGCACTGTGGAGATGAAC-3'; ApoB, forward, 5'-GAGCCTCTAATTTTGCTGGG-3' and reverse 5'-TGTTCCCATGTGCCATAGAT-3'; LDLR, forward 5'-AAGGCTGTGGGTTCCATAGG-3'and reverse 5'-TGGACCCTTTCTCTCGGAAC-3'; and $\beta$-actin, forward 5'- GCTAACAGTCCGCCTAGAAGCA-3' and reverse 5'-GTCATCACCATCGGCAATGAG-3'. The qPCR conditions were: one cycle at $95^{\circ} \mathrm{C}$ for $5 \mathrm{~min} ; 40$ cycles at $95^{\circ} \mathrm{C}$ for $15 \mathrm{sec}$ and $60^{\circ} \mathrm{C}$ for $60 \mathrm{sec}$; one cycle at $95^{\circ} \mathrm{C}$ for $15 \mathrm{sec}, 60^{\circ} \mathrm{C}$ for $1 \mathrm{~min}$, and $95^{\circ} \mathrm{C}$ for $15 \mathrm{sec}$. Thermal cycling and real-time detection were conducted on a StepOnePlus Real-Time PCR System (Applied Biosystems; Thermo Fisher Scientific, Inc.). Relative fold changes in mRNA expression were calculated using the formula $2^{-\Delta \Delta \mathrm{Cq}}(23)$.

Western blot analysis. A bicinchoninic acid kit (Sigma-Aldrich; Merck KGaA) was used to determine the protein concentration of liver samples from five rats per group. Sample buffer (SDS-PAGE sample buffer; Beyotime Institute of Biotechnology) was added, and the samples were boiled at $95^{\circ} \mathrm{C}$ for $10 \mathrm{~min}$. Next, proteins $(30 \mu \mathrm{g}$ per sample, per well) were separated by $10 \%$ polyacrylamide gel electrophoresis. The proteins were transferred to polyvinylidene fluoride membranes by $100 \mathrm{~V}$ transfer-molded voltage for 45 to $70 \mathrm{~min}$. The samples were incubated at room temperature for $1 \mathrm{~h}$ with $5 \%$ bovine serum albumin, and subsequently with rabbit anti-MTTP (cat. no. PA5-42391), rabbit anti-ApoB (cat. no. PA5-114864), rabbit anti-LDLR (cat. no. PA5-82385) and mouse anti- $\beta$-actin primary antibodies (cat. no. MA5-15739-D550; all used at 1:1,000 dilution; all supplied from Thermo Fisher Scientific, Inc.), at $4^{\circ} \mathrm{C}$ overnight. Next, the samples were washed three times in Tris-buffered saline/0.05\% Tween-20 (5 min each wash). The corresponding HRP-conjugated secondary antibody (cat. no. 21130; 1:20,000 dilution; Thermo Fisher Scientific, Inc.) was added and incubated at room temperature for $1 \mathrm{~h}$. The membranes were washed three times ( 5 min each wash). Bands were developed using chemiluminescence reagents; $\beta$-actin was used as the internal reference. Bands were visualized with a Bio-Rad Gel Doc EZ Imager (Bio-Rad Laboratories, Inc.) and band intensity was analyzed using ImageJ software (Version 1.53; National Institutes of Health).

Statistical analysis. Data were analyzed using Prism version 6 (GraphPad Software, Inc.) statistical software. Each experiment was repeated three times. Data are expressed as means \pm standard deviation. Kruskal-Wallis followed by Dunn's test was used to assess NAS scores. One-way analysis
Table I. Feed composition.

A, Normal diet

\begin{tabular}{lr}
\hline Ingredient & Content $(\%)$ \\
\hline Nitrogen-free extract & 44.72 \\
Fat & 5.34 \\
Crude protein & 23.82 \\
Crude ash & 6.18 \\
Crude fiber & 2.82 \\
Crude fat & 6.16 \\
Water & 8.96 \\
Calcium & 1.17 \\
Phosphorus & 0.83 \\
\hline
\end{tabular}

B, High-fat diet

Ingredient

Content $(\%)$

Normal diet

80

Yolk powder

8

Lard oil

10

Cholesterol

1.5

Sodium cholate

0.5

of variance followed by LSD post hoc test was applied for comparisons of multiple groups. $\mathrm{P}<0.05$ was considered to indicate a statistically significant difference.

\section{Results}

General observation. The rats in the ND group were active, responsive, had a good appetite, and were clean and shiny, without hair loss. Their stools were granular, yellow, and hard. In the HFD group, the rats were obese, unresponsive, and their hair was yellow and dull. Hair loss occurred with time, appetite decreased, and stools were granular, black, and hard. Following administration of BBR, the rats exhibited fur that was less yellow, reduced hair loss, they were more responsive, and their feces were watery.

Body weight and liver wet weight. As shown in Fig. 1, the body weight and liver wet weight of HFD rats increased significantly compared with the ND group $(\mathrm{P}<0.05)$. The body weight and liver wet weight of the BBR group were significantly lower compared with the HFD group $(\mathrm{P}<0.05$; Fig. 1).

Serum biochemical indices. Long-term feeding of a high-fat diet resulted in higher levels of ALT, AST, TG, TC, FBG, and LDL in the HFD group compared with the ND group, and significantly lower HDL concentrations (all $\mathrm{P}<0.05$; Table II). After BBR intervention, serum ALT, AST, TG, TC and LDL decreased significantly compared with the HDF group $(\mathrm{P}<0.05$; Table II). Notably, the serum levels of ALT, TG, TC and LDL were similar to those of the ND group, suggesting that BBR treatment successfully reversed these biochemical 
Table II. Serum biochemical parameters.

\begin{tabular}{|c|c|c|c|c|c|c|c|}
\hline Group & $\begin{array}{l}\text { ALT } \\
(\mathrm{U} / 1)\end{array}$ & $\begin{array}{l}\text { AST } \\
(\mathrm{U} / 1)\end{array}$ & $\begin{array}{c}\mathrm{TG} \\
(\mathrm{mol} / \mathrm{l})\end{array}$ & $\begin{array}{c}\mathrm{TC} \\
(\mathrm{mmol} / \mathrm{l})\end{array}$ & $\begin{array}{c}\text { FBG } \\
(\mathrm{mmol} / \mathrm{l})\end{array}$ & $\begin{array}{c}\text { LDL } \\
(\mathrm{mmol} / \mathrm{l})\end{array}$ & $\begin{array}{c}\text { HDL } \\
(\mathrm{mmol} / \mathrm{l})\end{array}$ \\
\hline ND & $49.63 \pm 4.21$ & $120.19 \pm 10.36$ & $1.57 \pm 0.16$ & $1.74 \pm 0.09$ & $5.19 \pm 0.34$ & $0.63 \pm 0.08$ & $1.19 \pm 0.08$ \\
\hline HFD & $96.24 \pm 10.11^{\mathrm{a}}$ & $173.20 \pm 15.46^{\mathrm{a}}$ & $2.10 \pm 0.19^{\mathrm{a}}$ & $2.33 \pm 0.18^{\mathrm{a}}$ & $6.30 \pm 0.61^{\mathrm{a}}$ & $1.97 \pm 0.15^{\mathrm{a}}$ & $0.57 \pm 0.05^{\mathrm{a}}$ \\
\hline BBR & $53.17 \pm 5.35^{\mathrm{b}}$ & $136.51 \pm 15.34^{\mathrm{a}, \mathrm{b}}$ & $1.69 \pm 0.09^{b}$ & $1.85 \pm 0.14^{\mathrm{b}}$ & $5.78 \pm 0.50^{\mathrm{a}}$ & $0.73 \pm 0.13^{b}$ & $0.64 \pm 0.08^{\mathrm{a}}$ \\
\hline
\end{tabular}

${ }^{\mathrm{a}} \mathrm{P}<0.05$ vs. ND; ${ }^{\mathrm{b}} \mathrm{P}<0.05$ vs. HFD. ALT, alanine aminotransferase; AST, aspartate aminotransferase; TG, triglyceride; TC, total cholesterol; FBG, fasting blood glucose; LDL, low-density lipoprotein; HDL, high-density lipoprotein; ND, normal diet; HFD, high-fat diet; BBR, berberine.

A

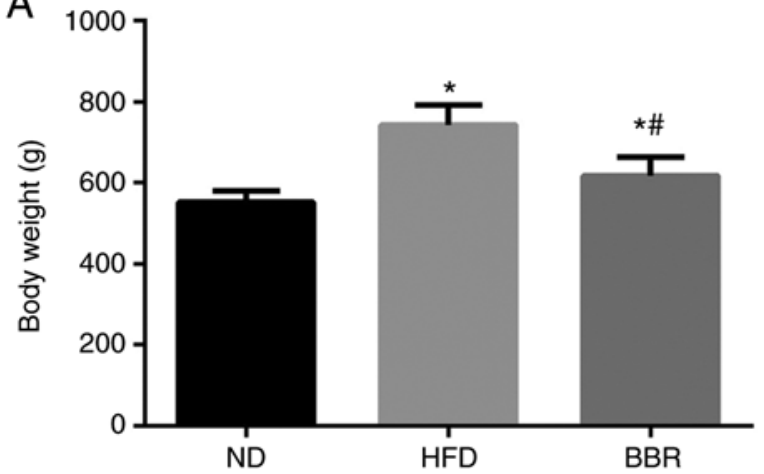

B

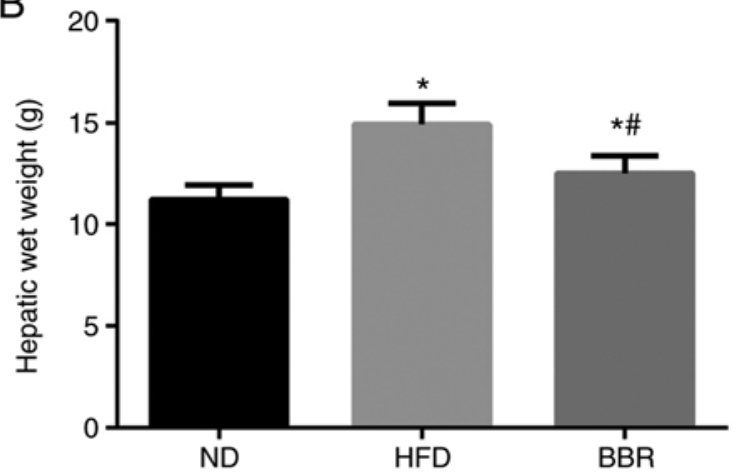

Figure 1. Body weight and hepatic wet weight. (A) Total body weight and (B) liver wet weight were measured in the rats of the ND, HFD and BBR groups before sample collection. ${ }^{*} \mathrm{P}<0.05$ compared with the ND group, ${ }^{\#} \mathrm{P}<0.05$ compared with the HFD group. ND, normal diet; HFD, high-fat diet; BBR, berberine.

indices $(\mathrm{P}>0.05$; Table II). No effect was observed after BBR administration for the serum levels of FBG and HDL, with their levels being similar in the BBR and HFD groups (Table II).

BBR alleviates liver damage induced by a high-fat diet. $H \& E$ staining revealed that the liver cells of the ND group were arranged neatly and tightly, the hepatic lobule structure was intact, the nucleus was located in the center of hepatocytes, the cytoplasm was uniform, and no lipid droplets were deposited (Fig. 2A). In the HFD group, the hepatocyte volume was increased, the structure of the hepatic lobule was incomplete, and there was a large amount of vacuolar-like steatosis (Fig. 2A). In severe cases, the cytoplasm exhibited a fishnet-like change. Following BBR treatment, vacuolar-like steatosis was reduced compared with the HFD group, and hepatocytes were arranged neatly (Fig. 2A). The NAS of the $\mathrm{ND}, \mathrm{HFD}$ and BBR groups was 0, $657 \pm 0.87$, and 2.55 \pm 0.56 , respectively (Fig. 2B), indicating that BBR significantly reduced NAS. These findings suggested that BBR administration alleviated liver damage.

A high-fat diet results in hepatic lipid deposition. The liver TG content was significantly higher in the HFD group compared with the ND group (Fig. 3). BBR administration significantly reduced the liver TG content $(\mathrm{P}<0.05$; Fig. 3$)$, consistent with the liver pathology results.

Expression levels of MTTP, ApoB, and LDLR. Next, the mRNA and protein expression levels of MTTP, ApoB, and
LDLR were determined in the liver. As shown in Fig. 4A, the mRNA expression levels of MTTP and LDLR in the HFD group decreased significantly compared with the ND group $(\mathrm{P}<0.05)$, while the mRNA expression levels of ApoB did not significantly change $(\mathrm{P}>0.05)$. Following BBR administration, the mRNA expression levels of MTTP and LDLR were upregulated, albeit the change in LDLR levels was not significant ( $\mathrm{P}>0.05$; Fig. 4A). The mRNA expression levels of ApoB were significantly decreased in the BBR group compared with the $\mathrm{ND}$ and HFD groups $(\mathrm{P}<0.05$; Fig. 4A). Western blot analysis revealed similar trends at the protein level. The protein expression levels of MTTP and LDLR were significantly decreased in the HFD group compared with the ND group ( $\mathrm{P}<0.05$; Fig. $4 \mathrm{~B})$, but there was no significant difference in ApoB protein expression levels ( $\mathrm{P}>0.05$; Fig. 4B). BBR administration significantly increased MTTP protein expression levels compared with those in the HFD group ( $\mathrm{P}<0.05$; Fig. 4B), but the increase in LDLR protein levels was not significant ( $P>0.05$; Fig. 4B). Finally, ApoB protein expression levels were significantly decreased in the BBR group compared with the ND and HFD groups $(\mathrm{P}<0.05$; Fig. 4A).

\section{Discussion}

The global incidence of NAFLD is $20 \%$. Lifestyle changes have led to a growing incidence of NAFLD (24-27). NAFLD is a metabolic syndrome that is often accompanied by diabetes and hyperlipidemia. These diseases are closely related to lipid metabolism disorders and abnormal lipid deposition, and their prevention and treatment are a focus of interest. 


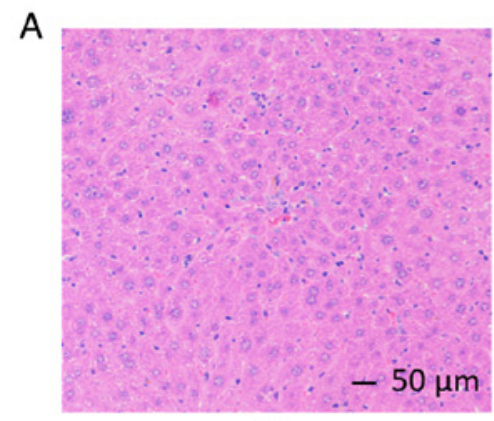

ND

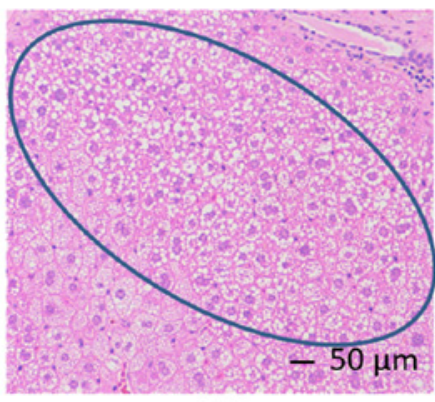

HFD

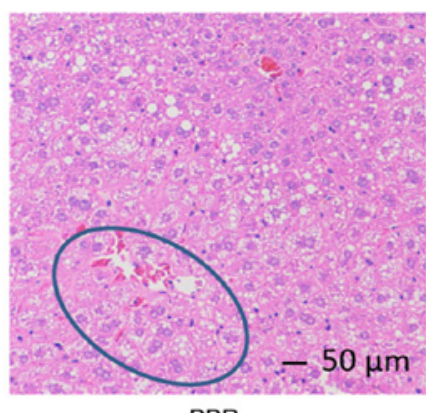

BBR

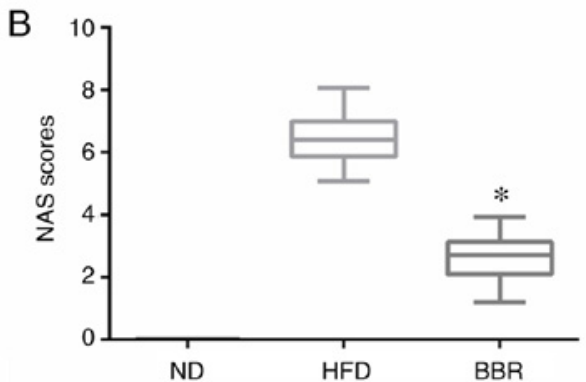

Figure 2. BBR alleviates liver damage. (A) Representative images from H\&E-stained liver sections showing that BBR administration alleviated liver damage induced by a high-fat diet. Abnormal cells are shown in circles. (B) NAS scores of ND, HFD and BBR groups. Higher scores mean increased severity. ${ }^{*} \mathrm{P}<0.05$ compared with the HFD group. BBR, berberine; NAS, nonalcoholic fatty liver disease activity score; ND, normal diet; HFD, high-fat diet.

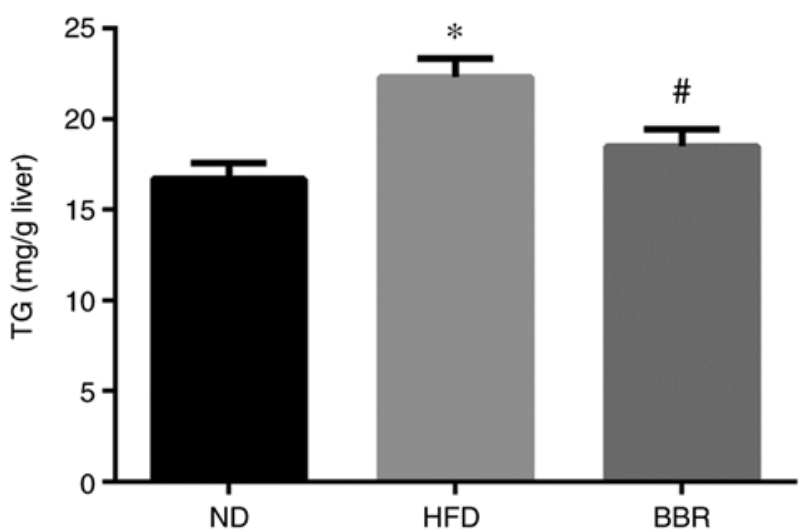

Figure 3. Effect of BBR on liver TG content. TG levels were measured in the livers from the rats in the three experimental groups. ${ }^{*} \mathrm{P}<0.05$ compared with the ND group; ${ }^{\#} \mathrm{P}<0.05$ compared with the HFD group. BBR, berberine; TG, triglyceride; ND, normal diet; HFD, high-fat diet.

The pathogenesis of NAFLD has been explained by first and second-strike theories. The second-strike theory proposed by Day and James (28) is generally accepted by the scientific community. The first strike refers to the rapid production of free fatty acids in the case of insulin resistance. The liver's ability to $\beta$-oxidize fatty acids and to synthesize VLDL decreases, leading to fat deposition in liver cells. The resulting imbalance of lipid metabolism triggers development of fatty liver. In the second strike, damaged hepatocytes result in increased levels of inflammatory factors and a reduced antioxidant capacity, which further aggravates fatty liver disease. In summary, the occurrence and development of NAFLD are closely related to lipid metabolism.

BBR has an anti-arrhythmia effect, dilates coronary arteries, induces hypoglycemia, and regulates blood lipids.
BBR has produced good results in clinical trials in type 2 diabetes with hyperlipidemia (29). Currently, there is no drug for NAFLD; because BBR has hypoglycemic, lipid-lowering, and antioxidant activities, the present study evaluated its effect on NAFLD. Yang et al (13) showed that BBR had a protective effect on NAFLD in animals. Kim et al (8) reported that BBR ameliorated lipid metabolism disorders in obese rats by interfering with the metabolism of surrounding tissues, thereby reducing liver wet weight and blood TG and TC levels. In the present study, BBR was administered to NAFLD rats to investigate its effect on NAFLD $(30,31)$. The mean body weight of the rats in the HFD group was significantly higher compared with that of rats in the ND group, and significantly reduced following BBR administration. Total visceral fat was increased in NAFLD rats. The mean liver wet weight was significantly higher in rats in the HFD group compared with those in the ND group. After 8 weeks of BBR administration, the liver wet weight of rats in the BBR group decreased significantly to a level similar to that of the ND group. AST and ALT are important functional enzymes in hepatocytes and are used as indicators of liver function. AST is mainly present in the myocardium, followed by the liver mitochondria. Under normal circumstances, serum ALT and AST levels are low. Damage to hepatocytes increases their membrane permeability, resulting in release of ALT and AST into plasma. In patients with hepatocyte injury, changes in ALT and AST levels reflect disease progression, thus facilitating clinical diagnosis. ALT and AST levels were significantly higher in the serum of the rats in the HFD group compared with the normal rats. The ALT and AST levels in those two groups were significantly lower than those in the HFD group after BBR administration. BBR may protect against liver damage caused by non-alcoholic factors by regulating ALT and AST, possibly by improving 
A

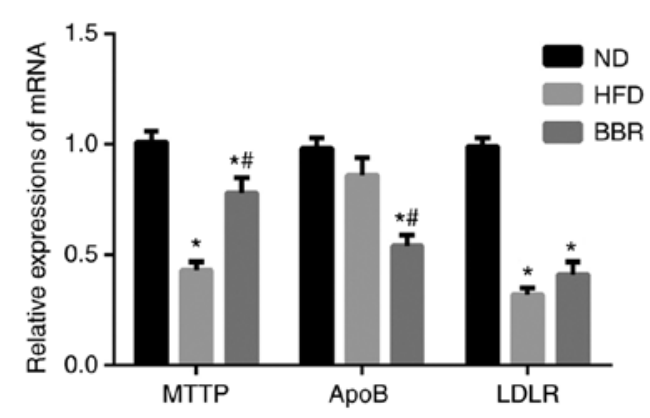

B

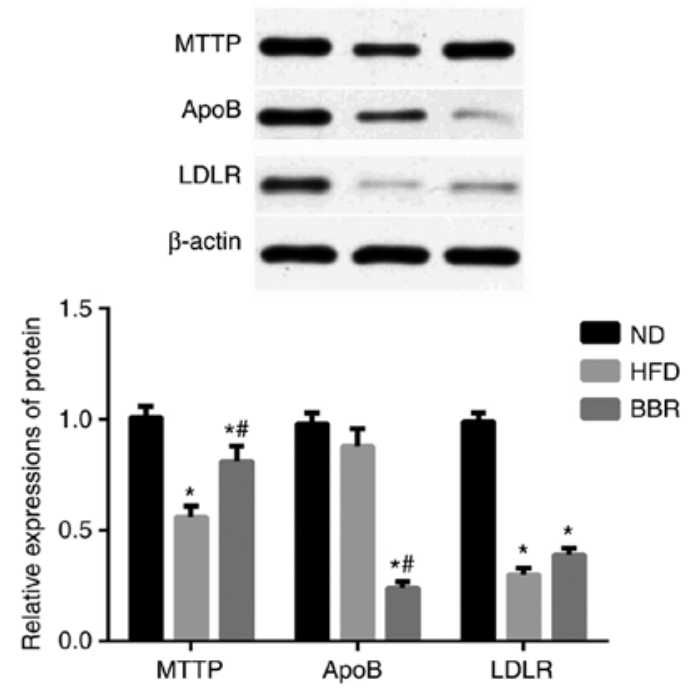

Figure 4. Effect of BBR on MTTP, ApoB and LDLR liver expression. (A) mRNA expression levels and (B) protein expression levels of MTTP, ApoB, and LDLR were measured in the livers from the rats in the three experimental groups. ${ }^{*} \mathrm{P}<0.05$ compared with the ND group; ${ }^{\sharp} \mathrm{P}<0.05$ compared with the $\mathrm{HFD}$ group. $\mathrm{BBR}$, berberine; MTTP, microsomal triglyceride transfer protein; ApoB, apolipoprotein B; LDLR, low-density lipoprotein receptor; ND, normal diet; HFD, high-fat diet.

the function of the liver cell membrane. NAFLD is associated with dyslipidemias such as hypertriglyceridemia, elevated LDL cholesterol, and decreased HDL cholesterol (32). HDL and LDL can be used to measure cholesterol carrying capacity. LDL is positively correlated with serum total cholesterol levels and it transports liver cholesterol to extrahepatic tissue cells. When the LDL concentration increases, LDL is deposited on arterial walls, forming an atherosclerotic plaque and blocking blood vessels. HDL is negatively correlated with total cholesterol levels. Its main role is to transport of extrahepatic cholesterol into liver cells for degradation and decomposition. The LDL and HDL levels reflect liver function. Accordingly, the present study demonstrated that rats fed a high-fat diet showed elevated serum TC and LDL levels and decreased HDL levels. BBR administration reduced serum $\mathrm{TC}$ and $\mathrm{LDL}$ levels and increased HDL levels. Continuous administration of BBR to rats for 8 weeks reduced liver TG content, significantly reversed hepatic steatosis, and enhanced liver function.

NAFLD features accumulation of large amounts of TG in the liver; this can be caused by diverse factors. Under normal circumstances, TG is excreted from the liver in the form of VLDL. Therefore, timely synthesis and secretion of VLDL is important for relieving TG deposition. The assembly and maturation of VLDL occur as follows (33-36): in the endoplasmic reticulum, ApoB forms under the action of MTTP, resulting in immature VLDL particles with lower precursor fat content, smaller particle size and higher density. The lipid droplets transfer large amounts of fat to immature VLDL particles, transforming them into higher fat, less dense, mature VLDL particles. An adequate fat supply and MTTP activity are necessary for VLDL synthesis. In the absence of sufficient fat supply or MTTP, ApoB cannot obtain fat, causing it to be degraded by the proteasome during or after translation (31). This prevents the production of VLDL particles to excrete TG. Wang et al (37) found that inhibition of MTTP activity impeded assembly of VLDL, leading to increased intrahepatic TG levels. It was hypothesized that the decreased expression of the MTTP gene in NAFLD rats may reduce TG excretion from the liver in VLDL, promoting hepatic fat deposition. BBR administration upregulated the expression and activity of MTTP, thereby enhancing the assembly of VLDL and reducing the liver TG content.

LDLR is a cell-surface glycoprotein that regulates plasma cholesterol levels by mediating LDL transport. Defects in LDLR function are one of the main causes of hypercholesterolemia, atherosclerosis and fatty liver (38). Current understanding of the role of LDLR in fatty liver has increased, and drugs that enhance LDLR expression to lower blood cholesterol levels have been developed. In the present study, BBR administration upregulated the expression of LDLR in NAFLD rats, suggesting that BBR may prevent fatty liver by regulating the LDLR content of hepatocytes and increasing the LDL clearance rate.

The second-strike is damage to cells by oxygen free radicals, causing lipid peroxidation, protein denaturation, damage to the cell membrane, and release of inflammatory mediators. These changes result in an impaired inflammatory response. BBR exerts an anti-inflammatory effect by inhibiting neutrophil chemotaxis, generation of oxygen free radicals, the activity of phospholipase A2 and reducing the tissue prostaglandin E2 content, thus reducing inflammatory damage. The anti-inflammatory effect of BBR also enhances superoxide dismutase (SOD) activity, inhibits the production of TNF- $\alpha$, improves ischemia reperfusion and reduces liver damage (39-42). However, further research into the mechanism by which BBR enhances SOD activity and inhibits TNF- $\alpha$ production is needed. As a bacteriostatic drug, BBR is used clinically mainly for intestinal infectious diseases and diarrhea. In vitro, BBR enhances the phagocytic capacity of leukocytes and the hepatic reticuloendothelial system, inhibits the growth of intestinal bacteria, reduces the release of bacterial endotoxins, and enhances the removal of endotoxin from serum. This decreases the intestinal inflammatory response, as well as the release of inflammatory mediators, such as TNF- $\alpha$ and IL-6. These effects reduce inflammatory damage 
to the liver and insulin resistance, thereby protecting the liver (43-46).

While displaying anti-inflammatory effects, BBR treatment also increases liver AMP-activated protein kinase (AMPK) phosphorylation (which leads to its activation). When activated, AMPK is capable of suppressing lipogenesis through phosphorylating and inactivating the lipogenic enzyme acetyl-CoA carboxylase (ACC). In addition, a decrease in the production of malonyl-CoA due to AMPK inhibition of ACC results in an increase in fatty acid oxidation via releasing the inhibitory effect of malonyl-CoA on carnitine palmitoyltransferase 1A. These combined effects of AMPK activation are considered to largely account for BBR actions on reducing hepatic steatosis as described by previous studies $(8,47-49)$.

In summary, the present study suggested that BBR may increase VLDL production by reversing the abnormal expression of key genes in lipid metabolism (MTTP and LDLR), thereby improving the symptoms of NAFLD. A limitation of the current results is that only one dose of BBR was used based on previous literature; future studies further investigating additional doses and their effects will be required to fully elucidate the role of BBR in ameliorating NAFLD.

\section{Acknowledgements}

We thank Dr Wenxia Bai from Jiangsu Drug Safety Evaluation Center for performing the histopathological observation and assessment.

\section{Funding}

No funding was received.

\section{Availability of data and materials}

All data generated or analyzed during the present study are included in this published article.

\section{Authors' contributions}

PC, YL and LX performed the experiments, analyzed the data and wrote the paper. PC and LX designed the study and provided experimental materials, PC and LX confirmed the authenticity of all the raw data. All authors read and approved the final manuscript.

\section{Ethics approval and consent to participate}

All experimental procedures involving animals were approved by the Animal Experimental Ethics Committee of the Affiliated Hospital of Shandong Medical College (permit no. AEECAHSMC20205059).

\section{Patient consent for publication}

Not applicable

\section{Competing interests}

The authors declare that they have no competing interests.

\section{References}

1. Perumpail BJ, Khan MA, Yoo ER, Cholankeril G, Kim D and Ahmed A: Clinical epidemiology and disease burden of nonalcoholic fatty liver disease. World J Gastroenterol 23: 8263-8276, 2017.

2. Forbes S, Taylor-Robinson SD, Patel N, Allan P, Walker BR and Johnston DG: Increased prevalence of non-alcoholic fatty liver disease in European women with a history of gestational diabetes. Diabetologia 54: 641-647, 2011.

3. Chang Y, Jung HS, Yun KE, Cho J, Cho YK and Ryu S: Cohort study of non-alcoholic fatty liver disease, NAFLD fibrosis score, and the risk of incident diabetes in a Korean population. Am J Gastroenterol 108: 1861-1868, 2013.

4. Yamada T, Fukatsu M, Suzuki S, Wada T, Yoshida T and Joh T: Fatty liver predicts impaired fasting glucose and type 2 diabetes mellitus in Japanese undergoing a health checkup. J Gastroenterol Hepatol 25: 352-356, 2010.

5. Cohen JC, Horton JD and Hobbs HH: Human fatty liver disease: Old questions and new insights. Science 332: 1519-1523, 2011.

6. Fabbrini E, Sullivan S and Klein S: Obesity and nonalcoholic fatty liver disease: Biochemical, metabolic, and clinical implications. Hepatology 51: 679-689, 2010.

7. Sreenivasa Baba C, Alexander G, Kalyani B, Pandey R, Rastogi S, Pandey A and Choudhuri G: Effect of exercise and dietary modification on serum aminotransferase levels in patients with nonalcoholic steatohepatitis. J Gastroenterol Hepatol 21: 191-198, 2006.

8. Kim WS, Lee YS, Cha SH, Jeong HW, Choe SS, Lee MR, Oh GT, Park HS, Lee KU, Lane MD, et al: Berberine improves lipid dysregulation in obesity by controlling central and peripheral AMPK activity. Am J Physiol Endocrinol Metab 296: E812-E819, 2009.

9. Qian S, Ma L, Peng S, Xu Y, Wu K, Shen S, Zhang X, Sun Y and Ye J: ATP reduces mitochondrial MECR protein in liver of diet-induced obese mice in mechanism of insulin resistance. Biosci Rep 40: 40, 2020.

10. Qin S, Tang H, Li W, Gong Y, Li S, Huang J, Fang Y, Yuan W, Liu Y, Wang S, et al: AMPK and its activator berberine in the treatment of neurodegenerative diseases. Curr Pharm Des 26: 5054-5066, 2020.

11. Li Q, Zhao C, Zhang Y, Du H, Xu T, Xu X, Zhang J, Kuang T, Lai X, Fan G, et al: 1H NMR-based metabolomics coupled with molecular docking reveal the anti-diabetic effects and potential active components of berberis vernae on type 2 diabetic rats. Front Pharmacol 11: 932, 2020

12. Xing LJ, Zhang L, Liu T, Hua YQ, Zheng PY and Ji G: Berberine reducing insulin resistance by up-regulating IRS-2 mRNA expression in nonalcoholic fatty liver disease (NAFLD) rat liver. Eur J Pharmacol 668: 467-471, 2011.

13. Yang QH, Hu SP, Zhang YP, Xie WN, Li N, Ji GY, Qiao NL, Lin XF, Chen TY and Liu HT: Effect of berberine on expressions of uncoupling protein-2 mRNA and protein in hepatic tissue of non-alcoholic fatty liver disease in rats. Chin J Integr Med 17: 205-211, 2011.

14. Li S, Xu Y, Guo W, Chen F, Zhang C, Tan HY, Wang N and Feng Y: The impacts of herbal medicines and natural products on regulating the hepatic lipid metabolism. Front Pharmacol 11: 351, 2020.

15. Liu R, Wu K, Li Y, Sun R and Li X: Human antigen R: A potential therapeutic target for liver diseases. Pharmacol Res 155: 104684, 2020.

16. Li CH, Tang SC, Wong $\mathrm{CH}$, Wang Y, Jiang JD and Chen Y: Berberine induces miR-373 expression in hepatocytes to inactivate hepatic steatosis associated AKT-S6 kinase pathway. Eur J Pharmacol 825: 107-118, 2018.

17. Pan $X$ and Hussain MM: Diurnal regulation of microsomal triglyceride transfer protein and plasma lipid levels. J Biol Chem 282: 24707-24719, 2007.

18. Yuan F, Wang H, Tian Y, Li Q, He L, Li N and Liu Z: Fish oil alleviated high-fat diet-induced non-alcoholic fatty liver disease via regulating hepatic lipids metabolism and metaflammation: A transcriptomic study. Lipids Health Dis 15: 20, 2016.

19. Lu Z, He B, Chen Z, Yan M and Wu L: Anti-inflammatory activity of berberine in non-alcoholic fatty liver disease via the Angpt12 pathway. BMC Immunol 21: 28, 2020.

20. Mai W, Xu Y, Xu J, Zhao D, Ye L, Yu G, Wang Z, Lu Q, Lin J, Yang T, et al: Berberine inhibits nod-like receptor family pyrin domain containing 3 inflammasome activation and pyroptosis in nonalcoholic steatohepatitis via the ROS/TXNIP axis. Front Pharmacol 11: 185, 2020. 
21. Zhu X, Bian H, Wang L, Sun X, Xu X, Yan H, Xia M, Chang X, $\mathrm{Lu} \mathrm{Y,} \mathrm{Li} \mathrm{Y,} \mathrm{et} \mathrm{al:} \mathrm{Berberine} \mathrm{attenuates} \mathrm{nonalcoholic} \mathrm{hepatic}$ steatosis through the AMPK-SREBP-1c-SCD1 pathway. Free Radic Biol Med 141: 192-204, 2019.

22. Bedossa P: Pathology of non-alcoholic fatty liver disease. Liver Int 37 (Suppl 1): 85-89, 2017.

23. Livak KJ and Schmittgen TD: Analysis of relative gene expression data using real-time quantitative PCR and the 2(-Delta Delta C(T)) Method. Methods 25: 402-408, 2001

24. Kojima S, Watanabe N, Numata M, Ogawa T and Matsuzaki S Increase in the prevalence of fatty liver in Japan over the past 12 years: Analysis of clinical background. J Gastroenterol 38: 954-961, 2003.

25. Adams LA, Angulo $P$ and Lindor KD: Nonalcoholic fatty liver disease. CMAJ 172: 899-905, 2005

26. Bedogni G, Miglioli L, Masutti F, Tiribelli C, Marchesini G and Bellentani S: Prevalence of and risk factors for nonalcoholic fatty liver disease: The Dionysos nutrition and liver study. Hepatology 42: 44-52, 2005.

27. Browning JD, Szczepaniak LS, Dobbins R, Nuremberg P, Horton JD, Cohen JC, Grundy SM and Hobbs HH: Prevalence of hepatic steatosis in an urban population in the United States: Impact of ethnicity. Hepatology 40: 1387-1395, 2004.

28. Day CP and James OF: Steatohepatitis: A tale of two 'hits'? Gastroenterology 114: 842-845, 1998.

29. Kong W, Wei J, Abidi P, Lin M, Inaba S, Li C, Wang Y, Wang Z, $\mathrm{Si}$ S, Pan H, et al: Berberine is a novel cholesterol-lowering drug working through a unique mechanism distinct from statins. Nat Med 10: 1344-1351, 2004.

30. Adams LA, Lymp JF, St Sauver J, Sanderson SO, Lindor KD, Feldstein A and Angulo P: The natural history of nonalcoholic fatty liver disease: A population-based cohort study. Gastroenterology 129: 113-121, 2005.

31. Gibbons GF: Assembly and secretion of hepatic very-low-density lipoprotein. Biochem J 268: 1-13, 1990.

32. Gibbons GF, Wiggins D, Brown AM and Hebbachi AM: Synthesis and function of hepatic very-low-density lipoprotein. Biochem Soc Trans 32: 59-64, 2004.

33. Rutledge AC, Su Q and Adeli K: Apolipoprotein B100 biogenesis: A complex array of intracellular mechanisms regulating folding, stability, and lipoprotein assembly. Biochem Cell Biol 88: 251-267, 2010.

34. Fisher EA and Ginsberg HN: Complexity in the secretory pathway: The assembly and secretion of apolipoprotein B-containing lipoproteins. J Biol Chem 277: 17377-17380, 2002.

35. Olofsson SO, Stillemark-Billton P and Asp L: Intracellular assembly of VLDL: Two major steps in separate cell compartments. Trends Cardiovasc Med 10: 338-345, 2000.

36. Zhou M, Fisher EA and Ginsberg HN: Regulated Co-translational ubiquitination of apolipoprotein B100. A new paradigm for proteasomal degradation of a secretory protein. J Biol Chem 273 : 24649-24653, 1998.

37. Wang Y,Tran K and Yao Z: The activity of microsomal triglyceride transfer protein is essential for accumulation of triglyceride within microsomes in McA-RH7777 cells. A unified model for the assembly of very low density lipoproteins. J Biol Chem 274 27793-27800, 1999.
38. Furbee JW Jr, Francone O and Parks JS: In vivo contribution of LCAT to apolipoprotein B lipoprotein cholesteryl esters in LDL receptor and apolipoprotein E knockout mice. J Lipid Res 43: 428-437, 2002

39. Zou K, Li Z, Zhang Y, Zhang HY, Li B, Zhu WL, Shi JY, Jia Q and Li YM: Advances in the study of berberine and its derivatives: A focus on anti-inflammatory and anti-tumor effects in the digestive system. Acta Pharmacol Sin 38: 157-167, 2017.

40. Yan YQ, Fu YJ, Wu S, Qin HQ, Zhen X, Song BM, Weng YS, Wang PC, Chen XY and Jiang ZY: Anti-influenza activity of berberine improves prognosis by reducing viral replication in mice. Phytother Res 32: 2560-2567, 2018.

41. Warowicka A, Nawrot R and Goździcka-Józefiak A: Antiviral activity of berberine. Arch Virol 165: 1935-1945, 2020.

42. Wu X, Li X, Dang Z and Jia Y: Berberine demonstrates anti-inflammatory properties in Helicobacter pylori-infected mice with chronic gastritis by attenuating the Th17 response triggered by the B cell-activating factor. J Cell Biochem 119: 5373-5381, 2018.

43. Li CL, Tan LH, Wang YF, Luo CD, Chen HB, Lu Q, Li YC, Yang XB, Chen JN, Liu YH, et al: Comparison of anti-inflammatory effects of berberine, and its natural oxidative and reduced derivatives from Rhizoma Coptidis in vitro and in vivo. Phytomedicine 52: 272-283, 2019.

44. Chao G, Ye F, Yuan Y and Zhang S: Berberine ameliorates non-steroidal anti-inflammatory drugs-induced intestinal injury by the repair of enteric nervous system. Fundam Clin Pharmacol 34: 238-248, 2020

45. Cicero AF and Baggioni A: Berberine and its role in chronic disease. Adv Exp Med Biol 928: 27-45, 2016.

46. Wang K, Feng X, Chai L, Cao S and Qiu F: The metabolism of berberine and its contribution to the pharmacological effects. Drug Metab Rev 49: 139-157, 2017.

47. Zang M, Zuccollo A, Hou X, Nagata D, Walsh K, Herscovitz H, Brecher P, Ruderman NB and Cohen RA: AMP-activated protein kinase is required for the lipid-lowering effect of metformin in insulin-resistant human HepG2 cells. J Biol Chem 279: 47898-47905, 2004.

48. Brusq J-M, Ancellin N, Grondin P, Guillard R, Martin S, Saintillan $\mathrm{Y}$ and Issandou M: Inhibition of lipid synthesis through activation of AMP kinase: An additional mechanism for the hypolipidemic effects of berberine. J Lipid Res 47: 1281-1288, 2006.

49. Assifi MM, Suchankova G, Constant S, Prentki M, Saha AK and Ruderman NB: AMP-activated protein kinase and coordination of hepatic fatty acid metabolism of starved/carbohydrate-refed rats. Am J Physiol Endocrinol Metab 289: E794-E800, 2005.

(7) $($ ) This work is licensed under a Creative Commons Attribution-NonCommercial-NoDerivatives 4.0 International (CC BY-NC-ND 4.0) License. 